\title{
Quality of survival the 1st year with glioblastoma: a longitudinal study of patient-reported quality of life
}

\author{
Lisa Millgård Sagberg, MSc, ${ }^{1-3}$ Ole Solheim, MD, PhD, ${ }^{1-3}$ and Asgeir S. Jakola, MD, PhD ${ }^{1,4}$ \\ 1Department of Neurosurgery, St. Olavs University Hospital; ${ }^{2}$ Department of Neuroscience, Norwegian University of Science and \\ Technology; ${ }^{3}$ National Competence Centre for Ultrasound and Image-Guided Therapy, Trondheim, Norway; and ${ }^{4}$ Sahlgrenska \\ University Hospital, Department of Neurosurgery, Gothenburg, Sweden
}

\begin{abstract}
OBJECTIVE By exploring longitudinal patient-reported health-related quality of life (HRQoL), the authors sought to assess the quality of survival for patients in the 1 st year after diagnosis of glioblastoma.

METHODS Thirty unselected patients $\geq 18$ years who underwent primary surgery for glioblastoma in the period 20112013 were included. Using the generic HRQoL questionnaire EQ-5D 3L, baseline HRQoL was assessed before surgery and at postoperative follow-up after 1, 2, 4, 6, 8, 10, and 12 months.

RESULTS There was an apparent correlation between deterioration in HRQoL scores and tumor progression. Patients with permanent deterioration in $\mathrm{HRQOL}$ early after surgery represented a subgroup with rapid progression and short survival. Both positive and negative changes in HRQoL were more often seen after surgery than after radio- or chemotherapy. Patients with gross-total resection (GTR) reported better and more stable HRQoL. In a multivariable analysis preoperative cognitive symptoms $(p=0.02)$, preoperative functional status $(p=0.03)$, and $G T R(p=0.01)$ were independent predictors of quality of survival (area under the curve for EQ-5D $3 \mathrm{~L}$ index values).

CONCLUSIONS The results indicate that progression-free survival is not only a surrogate marker for survival, but also for quality of survival. Quality of survival seems to be associated with GTR, which adds further support for opting for extensive resections in glioblastoma patients with good preoperative functional levels.

http://thejns.org/doi/abs/10.3171/2015.4.JNS15194
\end{abstract}

KEY WORDS brain tumor; glioblastoma; surgery; outcome; quality of life; extent of resection; oncology

$\mathrm{G}$ LIOBLASTOMA is the most common primary malignant brain tumor in adults. ${ }^{6}$ Apart from ensuring a histological diagnosis, the surgical aim is typically to achieve "maximal safe resection" to alleviate symptoms and prolong survival. ${ }^{22,34,39}$ Despite optimal adjuvant treatment with radiotherapy and temozolomide, $, 0,42$ prognosis remains poor. In population-based data, median survival is only 10 months, and long-term survival is rare..$^{32}$

Since current treatments are essentially palliative, potential benefits should be carefully weighed against risks of adverse effects, also with regard to the short term due to the aggressive course of the disease. However, the palliative setting is not readily reflected in the neurosurgical literature, where the focus traditionally has been on resection grades, progression-free survival, and overall survival, none providing any direct information on quality of survival. To elucidate the patients' health-related quality of life (HRQoL) during the disease, a patient-reported outcome (PRO) is a necessity. Although HRQoL is more often reported in the oncological literature, the impact of surgery is unclear since baseline preoperative data are usually missing.

We have previously demonstrated the devastating effect of surgically acquired deficits on HRQoL in glioma patients, ${ }^{17}$ and that perioperative loss of HRQoL is negatively associated with survival in glioblastoma. ${ }^{16}$ However, it is largely unknown how potentially important factors such as recovery of transient deficits, progression of disease, adverse effect of adjuvant therapy, or other significant medical events influence the HRQoL beyond the immediate postoperative period and during the course of the disease. In the present study, we focus on longitudinal patient-reported HRQoL with serial assessments from before primary surgery and up to 12 months after surgery. Our aim was to assess quality of survival by exploring the dynamics of HRQoL in the 1st year after diagnosis of glioblastoma.

ABBREVIATIONS ASA = American Society of Anesthesiologists; $\mathrm{AUC}=$ area under the curve; $\mathrm{CCl}=$ Charlson Comorbidity Index; EORTC $=$ European Organisation for Research and Treatment of Cancer; GTR = gross-total resection; HRQoL = health-related quality of life; KPS = Karnofsky Performance Status; LOCF = last-observationcarried-forward; $\mathrm{MCID}$ = minimal clinically important difference; $\mathrm{PRO}$ = patient-reported outcome; RANO = Response Assessment in Neuro-Oncology criteria; VAS = visual analog scale.

SUBMITTED January 27, 2015. ACCEPTED April 2, 2015.

INCLUDE WHEN CITING Published online October 2, 2015; DOI: 10.3171/2015.4.JNS15194. 


\section{Methods}

\section{Study Design and Study Population}

In a longitudinal descriptive and exploratory study, we included 30 unselected patients $\geq 18$ years who underwent primary surgery for glioblastoma at St. Olavs University Hospital, Trondheim, Norway, in the period from October 2011 through April 2013. A flowchart of the inclusion process is shown in Fig. 1. The histopathological diagnosis of glioblastoma was confirmed by a neuropathologist according to the current WHO classification. ${ }^{24}$

\section{Treatment}

The surgical procedure was either craniotomy with tumor resection, or biopsy only for histological confirmation. A neuronavigation system with $3 \mathrm{D}$ preoperative MRI and updated intraoperative 3D ultrasound volumes was routinely used..$^{14}$ Functional neuronavigation based on preoperative functional MRI or diffusion tensor imaging was used when deemed helpful. ${ }^{2}$ After surgery, the patients were referred to the oncological department for further treatment, most often in the form of radiotherapy with concomitant and adjuvant chemotherapy (temozolomide) according to the Stupp protocol. ${ }^{40}$ Some patients were later reoperated on due to relapse during follow-up.

\section{HRQoL Instrument}

To ensure compliance in unselected glioblastoma patients, we chose EQ-5D 3L, a simple and brief generic HRQoL questionnaire developed by the EuroQol Group. ${ }^{10}$ EQ-5D 3L consists of 2 parts: a descriptive system (Part I) and a visual analog scale (VAS) (Part II). Part I consists of 5 single-item dimensions including mobility, selfcare, usual activities, pain/discomfort, and anxiety/depression. Each dimension is divided into 3 levels of severity, i.e., "no problem," "slight problem," or "major problem," which generate 243 possible combinations of responses. Part II uses VAS to measure health status, ranging from worst imaginable health state to best imaginable health state. EQ-5D 3L can be presented as a health profile or as a single global health index with a weighted total value for HRQoL. EQ-5D 3L index value will range from -0.594 to 1 , where 1 corresponds to perfect health, and 0 to death; negative values are considered to be worse than death. EQ-5D 3L is widely used in a variety of health conditions and was tested and found valid for use in the Norwegian normal population..$^{27}$ We have recently demonstrated that the questionnaire is responsive in deteriorating glioma patients, with a minimal clinically important difference (MCID) value of $\pm 0.15 .{ }^{33}$

\section{Data Collection}

Patients provided written informed consent and completed the HRQoL questionnaire 1-3 days before surgery. Postoperative follow-up with longitudinal assessment of HRQoL were performed after 1, 2, 4, 6, 8, 10, and 12 months. The per protocol intention was to assess HRQoL every month to obtain short and regular assessment intervals, but this turned out to be too frequent for the patients. To assure compliance, follow-up was based on structured telephone interviews conducted by a study nurse, and efforts were made to reduce missing data. When the patients had apparent considerable cognitive impairments, or were otherwise too ill to answer (e.g., severe dysphasia), proxy ratings were used. The proxies were asked to assess the patient as they thought the patient would respond, i.e., from a proxy-patient perspective. ${ }^{30}$ Thirteen percent of all interviews were proxy assisted. Follow-up assessments were completed on schedule \pm 18 days, except for 3 cases (25, 25, and 29 days over time).

Preoperative symptoms, Charlson Comorbidity Index (CCI), ${ }^{7}$ postoperative complications, and new or worsened neurological deficits after surgery were retrospectively captured from electronic medical records. The deficits were considered as transient if they improved within 30 days after surgery, while persistent deficits extended more than 30 days. ${ }^{23}$ Karnofsky Performance Status (KPS), a widely used functional status, ${ }^{19}$ was scored by the operating surgeon just prior to surgery. Radio- and chemotherapy were registered at each assessment if it was initiated

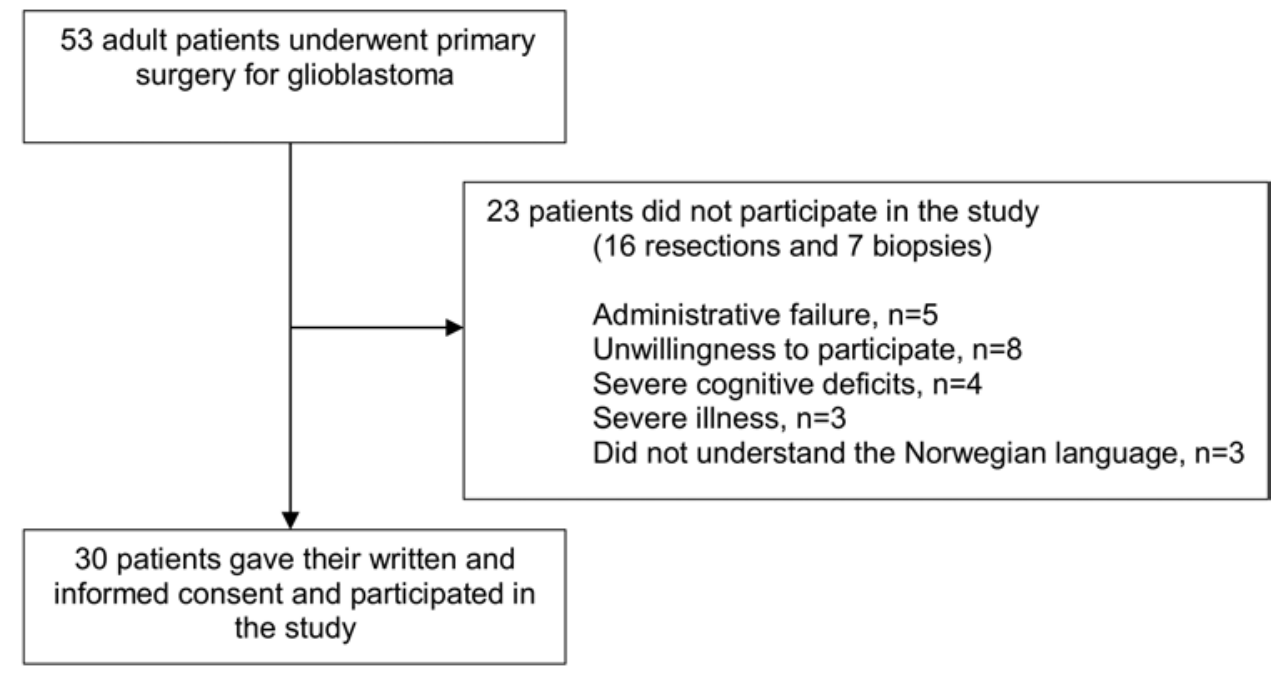

FIG. 1. Flowchart showing the inclusion process. 
or a new cycle had started since last follow-up. Tumor volumes were calculated by a neurosurgeon (A.J.), using an ellipsoid volume formula (4л $\times \mathrm{r}^{3} / 3$ ) based on the maximal tumor diameters in the perpendicular dimensions. ${ }^{38}$ In contrast-enhancing tumors (e.g., a typical glioblastoma with ring-like pattern of contrast enhancement), the contrast enhancement was used to calculate volumes. In glioblastomas with larger areas not exhibiting contrast enhancement, the entire volume as seen in T2/FLAIR sequences was used. The extent of resection was classified from preoperative and early postoperative ( $<48$ hours) MR images. In 1 patient without postoperative MRI, the resection grade was classified as subtotal after a review of the surgical report. Tumor progression, pseudoprogression, and complete response were determined according to the Response Assessment in Neuro-Oncology (RANO) criteria ${ }^{43}$ Eloquence was graded as suggested by Sawaya et al. ${ }^{35}$ Survival time was calculated as the time interval between surgery and death.

\section{Ethics and Approvals}

All patients involved in the study gave their written and informed consent. As the extensive data collection could be burdensome for severely ill patients, we treaded carefully by ensuring that interviews were ended if the patient seemed exhausted. Further, we asked all patients specifically if they wanted to participate in the next scheduled interview. Proxy ratings were used only in cases where the caregiver/next of kin agreed to respond. Data collection was approved by the Regional Committee for Medical and Health Research Ethics for Health Region Mid-Norway and adhered to the guidelines of the Helsinki Declaration. ${ }^{44}$

\section{Statistical Analyses}

All analyses were done using SPSS version 21.0 (IBM). Q-Q plots were used to test for normal distribution of data, and both simple descriptive analyses and hypothesis-generating exploratory analyses were carried out. EQ-5D 3L index scores were calculated according to the EuroQol scoring manual, using the UK time trade-off (TTO) value set. ${ }^{9}$ A heat map was created, in which we chose to dichotomize the summary scores to ease interpretation. The MCID level for the index score $( \pm 0.15)$ was used to group scores. ${ }^{33}$

The area under the curve (AUC), i.e., representing the cumulative quality of life in the 1st year of the disease, was calculated for each individual as described by Fayers and Machin. ${ }^{11}$ The EQ-5D 3L index value was set to equal death $(=0)$ if the patient died during the follow-up period. ${ }^{11}$ In a linear regression model, we tested variables possibly associated with AUC ( $p<0.10$ as univariables). Statistical significance level was set at $\mathrm{p}<0.05$.

\section{Missing Data}

In the heat map, missing data were imputed in 5\% of the HRQoL assessments. Last-and-next imputation was used in patients with available assessments before and after the missing assessment. Last-observation-carried-forward (LOCF) imputation was used in patients with no further assessments only if the disease was stable according to the RANO criteria. In cases with progressive disease, we left the data as missing due to an observed heterogeneous clinical course after progression.

In the AUC material, the overall imputation rate was $3 \%$. These calculations do not require regular intervals between follow-ups and could be carried out even when there was missing data. However, in cases where the last data point was missing in patients still alive $(n=4)$, imputation was done. In 1 case with progressive disease at 12 months, we calculated the mean decline in index value after progression in available patients $(-0.22)$ and subtracted this value from the last assessment. In 3 cases with missing forms and progressive disease, EQ-5D 3L index value was set to 0 at time of death (at 13, 15, and 17 months), and the gradual slope from last observation until 12 months was used in the equation. Also, to achieve trustworthy data in 2 cases with missing forms and stable disease, LOCF was imputed from last observation until progression.

\section{Results \\ Baseline Characteristics}

Preoperative data at baseline are summarized in Table 1. The mean age of patients was $62 \pm 11$ years, and 7 patients $(23 \%)$ were female. The patients presented a range of preoperative symptoms, and the most common were cognitive deficits, headache, seizures, and language deficits. A total of 23 patients (77\%) were functionally independent with a KPS $\geq 70$ prior to surgery.

TABLE 1. Baseline characteristics $(n=30)^{*}$

\begin{tabular}{|c|c|}
\hline Characteristic & Value \\
\hline Mean age in yrs \pm SD & $62 \pm 11$ \\
\hline Female & $7(23)$ \\
\hline \multicolumn{2}{|l|}{ Preop symptoms $\dagger$} \\
\hline Headache & $11(37)$ \\
\hline Seizures & $11(37)$ \\
\hline Cognitive symptoms & $15(50)$ \\
\hline Nausea & $2(7)$ \\
\hline Unsteadiness/ataxia & $9(30)$ \\
\hline Visual disturbances & $1(3)$ \\
\hline Aphasia/dysphasia & $12(40)$ \\
\hline Cranial nerve deficits & $2(7)$ \\
\hline Motor deficits & $5(17)$ \\
\hline \multicolumn{2}{|l|}{ Preop KPS } \\
\hline$\geq 90$ & $13(43)$ \\
\hline $70-80$ & $10(33)$ \\
\hline$\leq 60$ & $7(23)$ \\
\hline $\mathrm{CCl}>1$ & $5(17)$ \\
\hline \multicolumn{2}{|l|}{ ASA score } \\
\hline $1-2$ & $23(77)$ \\
\hline$\geq 3$ & $7(23)$ \\
\hline Multifocal & $7(23)$ \\
\hline Preop tumor volume in $\mathrm{cm}^{3}$, median (IQR) & $20(11-32)$ \\
\hline Eloquent location $\ddagger$ & $18(60)$ \\
\hline $\begin{array}{l}\text { IQR = interquartile range. } \\
\text { * Values are } n(\%) \text { except where indicated. } \\
\dagger \text { Multiple symptoms can occur simultaneously. } \\
\ddagger \text { In accordance with Sawaya et al. Grade } 3 .{ }^{35}\end{array}$ & \\
\hline
\end{tabular}




\section{Treatment and Disease Characteristics After Surgery}

As shown in Table 2, resection was performed in 24 patients (80\%). Median extent of resection was $98 \%$, with gross-total resection (GTR) achieved in 9 patients (30\%). Six patients (20\%), all with lesions in eloquent regions, acquired transient $(n=2)$ or persistent $(n=4)$ neurological deficits after surgery. Of these, only 1 patient suffered from a new deficit (motor) while the remaining 5 patients experienced worsening preexisting deficits. All 30 patients were treated with postoperative radiotherapy, all but 1 patient received concomitant temozolomide, and all but 4 were given adjuvant temozolomide. Tumor progression occurred in 25 patients $(83 \%)$, and 8 patients $(27 \%)$ underwent a second tumor resection within a year from primary surgery. At 12 months after surgery, 15 patients $(50 \%)$ had died.

\section{HRQoL During the 1st Year}

EQ-5D 3L index values, events, and survival for the individual patients are presented in a heat map (Fig. 2), where darker colors indicate lower HRQoL scores. As

TABLE 2. Treatment and disease characteristics $(n=30)^{*}$

\begin{tabular}{lc}
\hline \multicolumn{1}{c}{ Characteristic } & Value \\
\hline Resection grades & \\
Median extent of resection, $†$ \% (IQR) & $98(90-100)$ \\
GTR (100\%) & $9(30)$ \\
STR (1\%-99\%) & $15(50)$ \\
Biopsy only & $6(20)$ \\
\hline Neurological postop deterioration $\ddagger$ & $6(20)$ \\
Persistent new or increased motor deficit & $3(10)$ \\
Persistent increased cognitive deficit & $1(3)$ \\
Transient increased motor deficit & $1(3)$ \\
Transient SMA syndrome & $1(3)$ \\
\hline Postop complications§ & $5(17)$ \\
Cavity hematoma requiring evacuation & $1(3)$ \\
Osteomyelitis requiring surgery & $1(3)$ \\
UTI & $2(7)$ \\
Urinary retention requiring catheter & $1(3)$ \\
\hline Postop radiotherapy & $30(100)$ \\
Concomitant temozolomide & $29(97)$ \\
Adjuvant temozolomide & $26(87)$ \\
\hline Tumor progression within 1 yr & $25(83)$ \\
Reoperation within 1 yr & $8(27)$ \\
\hline Number of deaths within & \\
4 months & $1(3)$ \\
6 months & $5(17)$ \\
8 months & $6(20)$ \\
10 months & $11(37)$ \\
\hline 12 months & $15(50)$ \\
\hline
\end{tabular}

SMA = supplementary motor area; STR = subtotal resection; UTI = urinary tract infection.

* Values are $\mathrm{n}(\%)$ except where indicated.

† $\mathrm{N}=23$ due to biopsies excluded and 1 case (STR) without postoperative MRI.

$\ddagger$ If suffering a combined deficit the most severe is registered (each patient counted only once).

$\S$ Any complication registered within 30 days. If suffering several complications the most severe is registered. the colors represent absolute values, clinically important changes (being index value \pm 0.15 ) in the individual patients may differ, and discrepancies are marked in the heat map. The patients are grouped according to the extent of resection.

The dynamics of patient-reported HRQoL were heterogeneous, but some patterns can be recognized. The most striking pattern is the apparent correlation between tumor progression and deterioration in HRQoL scores. Of the 11 patients with a clinically important decline in HRQoL the 1 st month after surgery, 7 patients $(64 \%)$ did not recover. Instead these 7 individuals experienced rapid tumor progression and died within a year compared with the 1-year mortality rate of $35 \%$ in patients with only transient deterioration or stable/increased postoperative HRQoL. In the group with poor prognosis, preoperative cognitive symptoms (86\% vs 39\%), KPS < 90 (100\% vs 43\%), American Society of Anesthesiologists (ASA) score $\geq 3(43 \%$ vs $17 \%$ ), multifocal lesions ( $43 \%$ vs $17 \%$ ), and eloquence in location ( $71 \%$ vs $57 \%$ ) were more common, and fewer underwent GTR (14\% vs 35\%). There was no difference in median extent of resection (98\% in both groups), or administration of postoperative radio- and chemotherapy, but fewer went on with adjuvant chemotherapy (71\% vs $91 \%$ ) in the group that deteriorated.

A total of 9 patients (30\%) experienced adverse events from surgery within 30 days. Of these, 7 patients (78\%) did not report a postoperative reduction in HRQoL scores, but 2 patients with new worsened persistent motor deficits reported a permanent loss. At 2 months follow-up, 19 patients $(63 \%)$ had stable or increased HRQoL scores compared with baseline, and EQ-5D 3L index scores below 0.85 at this time point seems linked to progression and early death.

As seen in the heat map, the 9 patients with GTR reported a fairly stable HRQoL during the 1st year of the disease, a finding that may be linked to the delay of tumor progression. Also, their survival was longer (log-rank $\mathrm{p}<$ $0.01)$, and 7 of these patients (78\%) were still alive after 12 months, compared with only 8 (38\%) of 21 patients with subtotal resection or biopsy only. The 18 patients with lesions in eloquent areas reported worse overall HRQoL scores, and only 7 of these patients (39\%) were alive after 12 months. In contrast to the surgical treatment, postoperative radio- and chemotherapy did not appreciably influence the HRQoL.

\section{Predictors of Quality of Survival (Cumulative HRQoL Scores Over Time)}

We further explored possible predictors of cumulative HRQoL scores (AUC) during the 1st year. Age in years, preoperative tumor volume, EQ-5D 3L baseline value, $\mathrm{ASA} \geq 3$, and multifocal lesions were not significantly associated with AUC in univariable analyses ( $p>0.10)$. In the multivariable model (Table 3), we found independent associations between AUC and preoperative cognitive symptoms $(p=0.02)$, preoperative KPS $(p=0.03)$, and GTR $(p=0.01)$. The relationship between AUC and extent of resection are graphically presented in a box-plot (Fig. 3 ). The cumulative HRQoL is markedly higher in patients where GTR is achieved. 


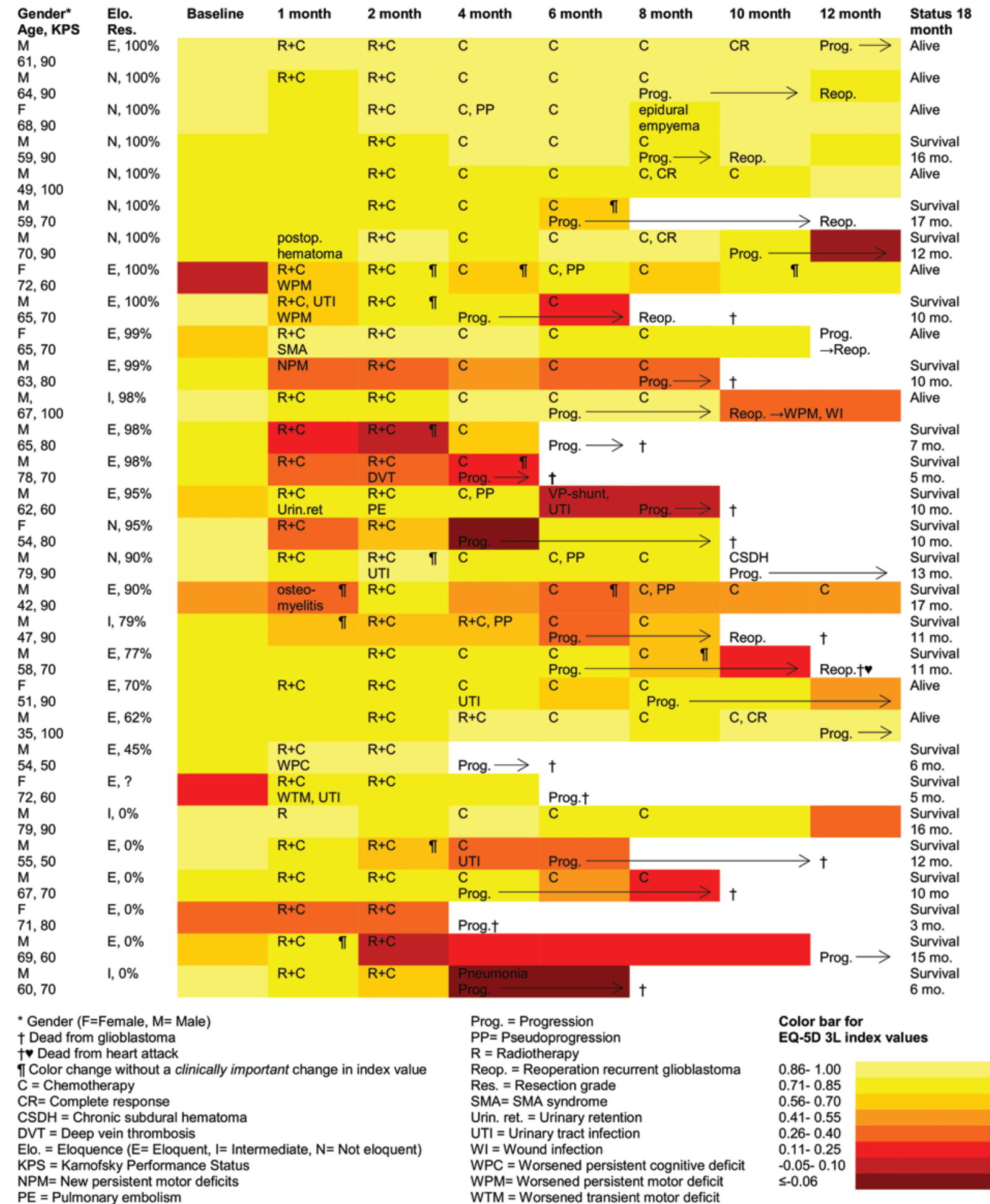

FIG. 2. Heat map showing the index values for EQ-5D 3L.

\section{Discussion}

\section{Main Findings}

In this longitudinal study of patient-reported HRQoL, we found that disease courses in newly diagnosed glioblastoma patients were heterogeneous. There was an ap- parent correlation between tumor progression and deterioration in HRQoL scores, and patients with persistent and progressive deterioration in HRQoL after surgery represented a subgroup with rapid progression and short survival. Both positive and negative changes in HRQoL were more often seen after surgery than after radio- or 
TABLE 3. Cumulative HRQoL and possible associated variables: linear regression analysis

\begin{tabular}{|c|c|c|c|c|}
\hline \multirow[b]{2}{*}{ Variable } & \multicolumn{2}{|c|}{ Univariate Analyses } & \multicolumn{2}{|c|}{ Multivariate Analyses } \\
\hline & OR $(95 \% \mathrm{Cl})$ & $p$ Value* & OR $(95 \% \mathrm{Cl})$ & $p$ Value* \\
\hline Preop cognitive symptoms & $-4.10(-6.24$ to -1.96$)$ & $<0.01$ & $-2.41(-4.34$ to -0.48$)$ & 0.02 \\
\hline Preop KPS & $0.15(0.08-0.22)$ & $<0.01$ & $0.09(0.01-0.16)$ & 0.03 \\
\hline GTR & $4.07(1.62-6.51)$ & $<0.01$ & $2.80(0.79-4.81)$ & 0.01 \\
\hline Eloquent locationt & $-2.78(-5.28$ to -0.28$)$ & 0.03 & $-0.18(-2.32$ to -1.96$)$ & 0.86 \\
\hline
\end{tabular}

chemotherapy. In patients where GTR was achieved, better and more stable HRQoL responses were reported. In a multivariable analysis, a high preoperative KPS score and GTR were independent predictors of better quality of survival in terms of cumulative HRQoL over 12 months, while preoperative cognitive symptoms was predictive of poorer quality of survival.

\section{Deterioration in HRQoL After Tumor Progression}

The deterioration in HRQoL following tumor progression is not surprising given the extensive symptom burden and fatal prognosis. In an earlier study by Osoba et al., HRQoL were assessed with the European Organisation for Research and Treatment of Cancer (EORTC) instruments QLQ-C30 and BN20, and lower functioning and more symptoms were found at recurrent disease compared with baseline..$^{29}$ However, their study was not longitudinal; hence, they could not provide any insight into when the decline in HRQoL occurred. Using a simple, generic HRQoL instrument, we were able to demonstrate that the decline in HRQoL from baseline to recurrence is not gradual but most often abrupt, around the time of progression. Thus, it does not seem unreasonable to claim that progression-free survival (here as defined by the RANO criteria) is not only a surrogate marker for survival but also for HRQoL. Our study also indicates that pseudoprogression is not similar-

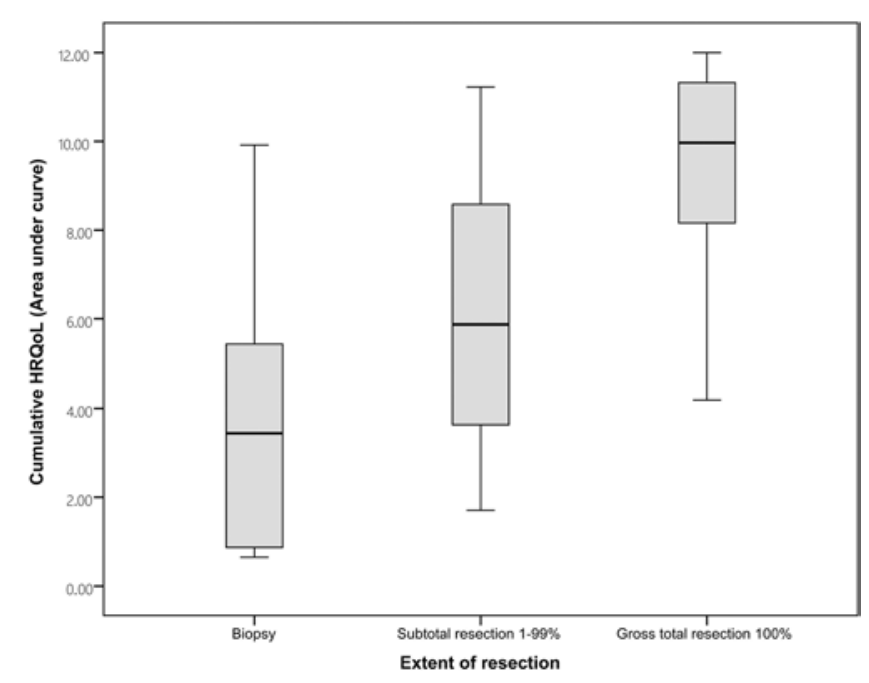

FIG. 3. Box plot showing the relationship between cumulative HRQoL and extent of resection. ly associated with deterioration in HRQoL, an important clinical finding given the difficulties with radiological assessment. The overall poor and rapidly declining HRQoL scores after tumor progression may limit the clinical potential of salvage therapy or second-line therapy for glioblastoma, at least if not given at the very first sign of tumor progression.

The single prior longitudinal HRQoL study that can be compared with the present study also found tumor progression to be associated with deterioration in HRQoL. ${ }^{45}$ However, that study by Yavas et al. excluded patients with KPS $<70$, and baseline HRQoL was assessed after surgery. The latter is important as we demonstrate that surgery is the therapy most likely to induce sudden significant positive or negative changes in HRQoL. Further, their assessments were more detailed but with longer intervals (3, $6,12,18$ months), making it difficult to establish exactly when the HRQoL changes occurred.

\section{HRQoL Changes After Surgery}

Changes in HRQoL were frequently seen after surgery, perhaps reflecting the potency both for symptom relief and for adverse effects. While patients with minor complications and transient deficits maintained their HRQoL, the 2 patients (7\%) with persistent motor deficits reported a permanent loss of HRQoL compared with baseline. Interestingly, both patients also had early progression and short survival, which indicates that the persistent decline in HRQoL scores may have had causes other than the adverse event per se. When adding this experience to our earlier demonstrated devastating effect of surgically acquired deficits on HRQoL in glioma patients, ${ }^{17}$ we believe that major and persistent deficits particularly reduce overall HRQoL during the 1st year. As functional status may be used for selecting treatment strategies, surgically acquired deficits may also prevent patients from receiving or completing oncological treatment. ${ }^{15}$

The perioperative HRQoL alterations may also reflect a more chaotic state of mind around the time of the glioblastoma diagnosis. Anxiety is often seen, and some patients may experience some relief after successful surgery, or when glucocorticoids are reduced or removed. The new situation will also disrupt normal life and affect the ability to perform usual daily activities, and for some individuals, there will be a shift in response over time as they adapt to their new circumstances.

Although previous studies have demonstrated that the Stupp regimen (i.e., radiotherapy with concomitant and 
adjuvant temozolomide) does not notably affect overall HRQoL, ${ }^{20,41}$ it may still cause a decline in HRQoL when there are adverse effects. ${ }^{40}$ We did not observe major changes in HRQoL around the time of radio- or chemotherapy administration, but our study was not particulary designed to detect adverse effects related to adjuvant treatment since the timing of HRQoL assessments were independent of treatment cycles and the sample size was small.

\section{Association Between Early Deterioration in HRQoL and Short Survival}

We have previously demonstrated that early deterioration in HRQoL following surgery is independently and markedly associated with impaired survival, ${ }^{16}$ and the same tendencies are seen in the present study. The rapid decline seen in some patients is seemingly not always due to perioperative complications, surgically induced deficits, or suboptimal extents of resection. As there is likely no negative selection bias in this study, it seems to us that as a consequence of inherent tumor biology, some glioblastomas rapidly progress despite maximal management effort. This is perhaps not surprising, but is still an important distinction to make when assessing perioperative HRQoL.

Even though HRQoL dynamics are not widely studied, it has previously been demonstrated that HRQoL may have prognostic capabilities. A meta-analysis of cancer patients demonstrated that HRQoL is an independent predictor of survival, ${ }^{31}$ and more specifically in neurooncology, an association has been found between fatigue and overall survival. ${ }^{4}$ However, a study involving 490 newly diagnosed glioblastoma patients concluded that baseline HRQoL, collected up to 8 weeks after surgery, added relatively little to known clinical factors for predicting survival. ${ }^{26} \mathrm{~A}$ major weakness of these studies is that baseline HRQoL was assessed after surgery. By including even short-term HRQoL dynamics (e.g., the time before surgery to end of radiotherapy) rather than a single static measure, more precise information may be achieved since early changes in HRQoL may reflect the aggressiveness of the disease. This was suggested by our previous study, ${ }^{16}$ and is confirmed in this study, where patients with early progressive deterioration after surgery had a substantially shorter survival. This is further supported by Bosma et al. ${ }^{3}$ who compared HRQoL in short-term and long-term survivors and found a deterioration in HRQoL in short-term survivals between baseline (after surgery, before radiotherapy) and 4 month follow-up, whereas the long-term survivors experienced improved HRQoL.

\section{Predictors of Cumulative HRQoL}

While there is a well-known association between GTR and survival in glioblastoma, ${ }^{22,34,39}$ we found in our present study that GTR is also associated with quality of survival. Without claiming causality from our observational data, our findings add support for opting for extensive resections in eligible glioblastoma patients, especially in patients with good preoperative functional levels. We also conclude that most patients with GTR have good overall HRQoL in the 1 st year after diagnosis, which can be of practical value when informing patients. The repeated HRQoL measurements not only capture the burden of disease or quality of survival, but presumably also increase statistical power compared with single outcome data. Simplified, detailed, and repeated data from fewer patients can match power in larger studies with coarse outcomes. Despite the limited number of patients, we were able to demonstrate seemingly independent effects of extent of resection (GTR) and patient selection (preoperative KPS and preoperative cognitive symptoms). In an earlier study, we did not observe any association between postoperative HRQoL or change in HRQoL and the extent of resection. ${ }^{17}$ This now stands in contrast to our findings in this study involving only 30 patients; the main reason for the difference may be due to the increased sensitivity of the instrument (EQ-5D 3L) when using cumulative HRQoL data.

Preoperative functional status is a well-known prognostic factor in glioblastoma patients,,$^{13,22}$ and in the present study, patients with better preoperative KPS also had better quality of survival in terms of cumulative HRQoL during the 1st year. The preoperative presence of cognitive symptoms was another independent predictor of poorer quality of survival. Cognitive impairment in newly diagnosed glioblastoma patients has consistently been associated with shorter survival. ${ }^{3,13,18,21}$ Whether cognitive function is a modifiable risk factor in glioblastoma is perhaps debatable. Although cognitive rehabilitation can be valuable for low-grade gliomas, we think it is not as feasible in high-grade gliomas with rapid tumor progression. Cognitive decline can clearly also be a function of more extensive disease and unfortunate localization of lesions. ${ }^{12}$ The poorer quality of survival in patients with cognitive symptoms and poorer functional status underlines the impact of patient selection for clinical results.

\section{Strengths and Weaknesses}

Previous studies evaluating the effect of glioblastoma surgery using PROs have early follow-up ${ }^{16,17}$ and/or have conducted complete data analysis, ${ }^{8,16,17}$ which may generate overoptimistic results since severely ill patients or patients with early deaths are not taken into account. Also, there are often strict inclusion criteria as part of oncological clinical trials, and patients with cognitive impairment ${ }^{8}$ or patients with KPS scores less than $70^{5,45}$ are often excluded. In the present study, we attempted to overcome these limitations. With the lack of controlled data for the assessment of efficacy of interventions, we believe the inclusion of an unselected population is a necessity to capture a true picture of the disease course. Our department serves a geographically defined catchment region, and we attempted to include consecutive unselected patients. However, as PRO studies require consent from patients, the inclusion of solely unselected patients is impossible. Still, we believe that the external validity of our findings are good given the population-based referral practice in Norway, the high inclusion rate, and the low drop-out rates.

We believe that the longitudinal design is a major strength since it made it possible to detect transient changes in HRQoL after surgery and to follow the disease course over time. Using cumulative HRQoL (AUC) is a novel approach in this context to quantify the quality of survival from the patients' perspectives. This is an attractive way to incorporate the aims of treatment, i.e., prolonging survival 
and maintaining/improving HRQoL into a single outcome measure. A similar approach was suggested for low-grade gliomas after the initiation of our study, ${ }^{25}$ but to our knowledge, no similar approaches have yet been presented for brain tumor patients. Using the approach from the lowgrade glioma study, attempting to define "time with quality of life", our present and previous results suggest that a score of 0.80-0.85 may be a reasonable threshold using EQ-5D 3L for glioblastomas. ${ }^{33}$

As missing data present a well-known challenge in HRQoL studies in general, and in end-of-life studies in particular, efforts were made to reduce this occurrence. The imputation rate was $\leq 5 \%$ and thereby low, ${ }^{36}$ and medical records were thoroughly read to secure that the imputations were as correct as possible. Thus, the data presented in this study are real-life, not modeled, data. Using proxy ratings was a necessity to include data for even the most dependent patients with cognitive deficits. Although this may have affected the answers to some extent, it is clearly better than having a lack of data. ${ }^{11,37}$

The major weakness in this study was the low sample size, which to some degree was compensated for with repeated measurements of HRQoL. Still, small patient samples are always vulnerable to bias due to chance, and as a consequence, we provide mainly descriptive data. Given the explorative nature of the study, our main objective was rather loosely defined as to simply present the HRQoL pattern over time and to generate hypotheses for further research.

EQ-5D 3L is a crude measure of HRQoL, and using a disease-specific HRQoL instrument would have been a preferable choice. For this reason, we intended to also use the EORTC QLQ-C30 and EORTC QLQ-BN20 instruments, ${ }^{1,28} 2$ frequently used tools in oncological brain tumor research. We soon realized that the original test regimen with monthly assessment became too tiring to patients. The short intervals were planned to have high temporal resolution to determine more precisely when changes in HRQoL occurred. Even though we changed to bimonthly assessments, extensive instruments containing 50 questions proved to be too burdensome and complicated for our unselected patients, and the rate of missing data became unacceptable. Instead, we analyzed and present only the EQ-5D 3L data. This generic and simple instrument, which also enabled proxy ratings, ensured an acceptable completeness of data, and we believe our findings strengthen the role for PRO assessment in surgical neuro-oncology.

\section{Conclusions}

In our novel study approach with longitudinal and cumulative HRQoL assessments in 30 unselected glioblastoma patients, we demonstrated that HRQoL most often seem to stabilize after surgery, until an abrupt rather than gradual decline occurs with tumor progression. This indicates that progression-free survival is not only a surrogate marker for survival but also for quality of survival. Patients with persistent and progressive decline in HRQoL early after surgery may represent a subgroup with especially poor prognosis despite active treatment. Independent predictors of better cumulative quality of survival in the 1st year after diagnosis were high preoperative KPS score and GTR, while preoperative cognitive symptoms was predictive of poorer quality of survival. This adds further support for opting for extensive resections in glioblastoma patients with good preoperative functional levels.

\section{Acknowledgments}

This work was supported by research grants from the Cancer Fund at St. Olavs University Hospital, Trondheim, Norway, and the Norwegian Cancer Society. We would like to thank Linda M. Nordtvedt at St. Olavs University Hospital for assisting in data collection.

\section{References}

1. Aaronson NK, Ahmedzai S, Bergman B, Bullinger M, Cull A, Duez NJ, et al: The European Organization for Research and Treatment of Cancer QLQ-C30: a quality-of-life instrument for use in international clinical trials in oncology. $\mathbf{J}$ Natl Cancer Inst 85:365-376, 1993

2. Berntsen EM, Gulati S, Solheim O, Kvistad KA, Torp SH, Selbekk T, et al: Functional magnetic resonance imaging and diffusion tensor tractography incorporated into an intraoperative 3-dimensional ultrasound-based neuronavigation system: impact on therapeutic strategies, extent of resection, and clinical outcome. Neurosurgery 67:251-264, 2010

3. Bosma I, Reijneveld JC, Douw L, Vos MJ, Postma TJ, Aaronson NK, et al: Health-related quality of life of long-term high-grade glioma survivors. Neuro Oncol 11:51-58, 2009

4. Brown PD, Ballman KV, Rummans TA, Maurer MJ, Sloan JA, Boeve BF, et al: Prospective study of quality of life in adults with newly diagnosed high-grade gliomas. J Neurooncol 76:283-291, 2006

5. Brown PD, Maurer MJ, Rummans TA, Pollock BE, Ballman $\mathrm{KV}$, Sloan JA, et al: A prospective study of quality of life in adults with newly diagnosed high-grade gliomas: the impact of the extent of resection on quality of life and survival. Neurosurgery 57:495-504, 2005

6. Central Brain Tumor Registry of the United States: CBTRUS Statistical Report: Primary Brain and Central Nervous System Tumors Diagnosed in the United States in 20042008. Hinsdale, IL: Central Brain Tumor Registry of the United States, 2012

7. Charlson ME, Pompei P, Ales KL, MacKenzie CR: A new method of classifying prognostic comorbidity in longitudinal studies: development and validation. J Chronic Dis 40:373383, 1987

8. Daigle K, Fortin D, Mathieu D, Saint-Pierre AB, Paré FM, de la Sablonnière A, et al: Effects of surgical resection on the evolution of quality of life in newly diagnosed patients with glioblastoma: a report on 19 patients surviving to follow-up. Curr Med Res Opin 29:1307-1313, 2013

9. Dolan P: Modeling valuations for EuroQol health states. Med Care 35:1095-1108, 1997

10. EuroQol Group: EuroQol--a new facility for the measurement of health-related quality of life. Health Policy 16:199208, 1990

11. Fayers PM, Machin D: Quality of Life: the Assessment, Analysis and Interpretation of Patient-Reported Outcomes, ed 2. Chichester, UK: Wiley, 2007

12. Gehring K, Sitskoorn MM, Gundy CM, Sikkes SA, Klein M, Postma TJ, et al: Cognitive rehabilitation in patients with gliomas: a randomized, controlled trial. J Clin Oncol 27:3712-3722, 2009

13. Gorlia T, van den Bent MJ, Hegi ME, Mirimanoff RO, Weller M, Cairncross JG, et al: Nomograms for predicting survival of patients with newly diagnosed glioblastoma: prognostic factor analysis of EORTC and NCIC trial 26981-22981/CE.3. Lancet Oncol 9:29-38, 2008

14. Gronningsaeter A, Kleven A, Ommedal S, Aarseth TE, Lie T, 
Lindseth F, et al: SonoWand, an ultrasound-based neuronavigation system. Neurosurgery 47:1373-1380, 2000

15. Gulati S, Jakola AS, Nerland US, Weber C, Solheim O: The risk of getting worse: surgically acquired deficits, perioperative complications, and functional outcomes after primary resection of glioblastoma. World Neurosurg 76:572-579, 2011

16. Jakola AS, Gulati S, Weber C, Unsgård G, Solheim O: Postoperative deterioration in health related quality of life as predictor for survival in patients with glioblastoma: a prospective study. PLoS One 6:e28592, 2011

17. Jakola AS, Unsgård G, Solheim O: Quality of life in patients with intracranial gliomas: the impact of modern image-guided surgery. J Neurosurg 114:1622-1630, 2011

18. Johnson DR, Sawyer AM, Meyers CA, O'Neill BP, Wefel JS: Early measures of cognitive function predict survival in patients with newly diagnosed glioblastoma. Neuro Oncol 14:808-816, 2012

19. Karnofsky DA, Burchenal JH: The clinical evaluation of chemotherapeutic agents in cancer, in MacLeod CM (ed): Evaluation of Chemotherapeutic Agents. New York: Columbia University Press, 1949

20. Keime-Guibert F, Chinot O, Taillandier L, Cartalat-Carel S, Frenay M, Kantor G, et al: Radiotherapy for glioblastoma in the elderly. N Engl J Med 356:1527-1535, 2007

21. Klein M, Postma TJ, Taphoorn MJB, Aaronson NK, Vandertop WP, Muller M, et al: The prognostic value of cognitive functioning in the survival of patients with high-grade glioma. Neurology 61:1796-1798, 2003

22. Lacroix M, Abi-Said D, Fourney DR, Gokaslan ZL, Shi W, DeMonte F, et al: A multivariate analysis of 416 patients with glioblastoma multiforme: prognosis, extent of resection, and survival. J Neurosurg 95:190-198, 2001

23. Landriel Ibañez FA, Hem S, Ajler P, Vecchi E, Ciraolo C, Baccanelli M, et al: A new classification of complications in neurosurgery. World Neurosurg 75:709-715, 604-611, 2011

24. Louis DN, Ohgaki H, Wiestler OD, Cavenee WK, Burger PC, Jouvet A, et al: The 2007 WHO classification of tumours of the central nervous system. Acta Neuropathol 114:97-109, 2007

25. Mandonnet E, Duffau H, Bauchet L: A new tool for grade II glioma studies: plotting cumulative time with quality of life versus time to malignant transformation. J Neurooncol 106:213-215, 2012

26. Mauer M, Stupp R, Taphoorn MJB, Coens C, Osoba D, Marosi $\mathrm{C}$, et al: The prognostic value of health-related quality-of-life data in predicting survival in glioblastoma cancer patients: results from an international randomised phase III EORTC Brain Tumour and Radiation Oncology Groups, and NCIC Clinical Trials Group study. Br J Cancer 97:302-307, 2007

27. Nord E: EuroQol: health-related quality of life measurement. Valuations of health states by the general public in Norway. Health Policy 18:25-36, 1991

28. Osoba D, Aaronson NK, Muller M, Sneeuw K, Hsu MA, Yung WK, et al: The development and psychometric validation of a brain cancer quality-of-life questionnaire for use in combination with general cancer-specific questionnaires. Qual Life Res 5:139-150, 1996

29. Osoba D, Brada M, Prados MD, Yung WK: Effect of disease burden on health-related quality of life in patients with malignant gliomas. Neuro Oncol 2:221-228, 2000

30. Pickard AS, Knight SJ: Proxy evaluation of health-related quality of life: a conceptual framework for understanding multiple proxy perspectives. Med Care 43:493-499, 2005

31. Quinten C, Coens C, Mauer M, Comte S, Sprangers MAG, Cleeland C, et al: Baseline quality of life as a prognostic indicator of survival: a meta-analysis of individual patient data from EORTC clinical trials. Lancet Oncol 10:865-871, 2009

32. Rønning PA, Helseth E, Meling TR, Johannesen TB: A population-based study on the effect of temozolomide in the treatment of glioblastoma multiforme. Neuro Oncol 14:1178-1184, 2012

33. Sagberg LM, Jakola AS, Solheim O: Quality of life assessed with EQ-5D in patients undergoing glioma surgery: what is the responsiveness and minimal clinically important difference? Qual Life Res 23:1427-1434, 2014

34. Sanai N, Berger MS: Glioma extent of resection and its impact on patient outcome. Neurosurgery 62:753-764, 264-266, 2008

35. Sawaya R, Hammoud M, Schoppa D, Hess KR, Wu SZ, Shi WM, et al: Neurosurgical outcomes in a modern series of 400 craniotomies for treatment of parenchymal tumors. Neurosurgery 42:1044-1056, 1998

36. Schulz KF, Grimes DA: Sample size slippages in randomised trials: exclusions and the lost and wayward. Lancet 359:781785,2002

37. Sneeuw KC, Sprangers MA, Aaronson NK: The role of health care providers and significant others in evaluating the quality of life of patients with chronic disease. J Clin Epidemiol 55:1130-1143, 2002

38. Stummer W, Pichlmeier U, Meinel T, Wiestler OD, Zanella F, Reulen HJ: Fluorescence-guided surgery with 5-aminolevulinic acid for resection of malignant glioma: a randomised controlled multicentre phase III trial. Lancet Oncol 7:392-401, 2006

39. Stummer W, Reulen HJ, Meinel T, Pichlmeier U, Schumacher W, Tonn JC, et al: Extent of resection and survival in glioblastoma multiforme: identification of and adjustment for bias. Neurosurgery 62:564-576, 2008

40. Stupp R, Mason WP, van den Bent MJ, Weller M, Fisher $\mathrm{B}$, Taphoorn MJ, et al: Radiotherapy plus concomitant and adjuvant temozolomide for glioblastoma. N Engl J Med 352:987-996, 2005

41. Taphoorn MJ, Stupp R, Coens C, Osoba D, Kortmann R, van den Bent MJ, et al: Health-related quality of life in patients with glioblastoma: a randomised controlled trial. Lancet Oncol 6:937-944, 2005

42. Wen PY, Kesari S: Malignant gliomas in adults. N Engl J Med 359:492-507, 2008

43. Wen PY, Macdonald DR, Reardon DA, Cloughesy TF, Sorensen AG, Galanis E, et al: Updated response assessment criteria for high-grade gliomas: response assessment in neurooncology working group. J Clin Oncol 28:1963-1972, 2010

44. World Medical Association: Declaration of Helsinki. Ferney-Voltaire, France: World Medical Association, 2008

45. Yavas C, Zorlu F, Ozyigit G, Gurkaynak M, Yavas G, Yuce $\mathrm{D}$, et al: Health-related quality of life in high-grade glioma patients: a prospective single-center study. Support Care Cancer 20:2315-2325, 2012

\section{Disclosure}

The authors report no conflict of interest concerning the materials or methods used in this study or the findings specified in this paper.

\section{Author Contributions}

Conception and design: Jakola. Acquisition of data: Sagberg. Analysis and interpretation of data: all authors. Drafting the article: Sagberg. Critically revising the article: all authors. Reviewed submitted version of manuscript: all authors. Approved the final version of the manuscript on behalf of all authors: Sagberg. Statistical analysis: all authors. Study supervision: Solheim, Jakola.

\section{Correspondence}

Lisa Millgård Sagberg, Department of Neurosurgery, St. Olavs University Hospital, Olav Kyrres gt 17, Trondheim 7006, Norway. email: lisa.millgard.sagberg@ntnu.no. 